\title{
Attribute-Based Clustering for Information Dissemination in Wireless Sensor Networks ${ }^{1}$
}

\author{
Ke Wang, Salma Abu Ayyash, Thomas D.C. Little \\ Department of Electrical and Computer Engineering \\ Boston University, Boston, Massachusetts 02215, USA \\ (617) 353-9877 \\ $\{k e, s a a y y a s h, t d c l\} @ b u . e d u$ \\ Prithwish Basu \\ BBN Technologies \\ 10 Moulton St, Cambridge, Massachusetts 02138, USA \\ pbasu@bbn.com
}

MCL Technical Report No. 07-13-2005

\begin{abstract}
Data routing in large wireless sensor networks is challenged by requirements for scalability, robustness, and energy use, as well as the possibility that globally unique identifiers for each node are nonexistent. Moreover, if the sensor network serves multiple functions or users, its routing function must be energy efficient when responding to information requests (inquiries) that can arrive at high rates, require diverse data types, and target subsets of the sensor network.

We propose virtual "containment" hierarchies based on relevant attributes as a more efficient mechanism to disseminate inquiries rather than the use of flooding schemes. We show algorithms that support clustering sensors according to these hierarchies and implement clusterhead failure recovery and load balancing among cluster members. We show that our framework has significant bandwidth gains over a flooding-based scheme under the scenario considered using analytical techniques.
\end{abstract}

\footnotetext{
${ }^{1}$ Proc. 2nd Annual IEEE Communications Society Conf. on Sensor and Ad Hoc Communications and Networks (SECON 2005), Santa Clara, CA, Sept. 2005. This work is supported by the NSF under grant No. CNS-0435353. Any opinions, findings, and conclusions or recommendations expressed in this material are those of the author(s) and do not necessarily reflect the views of the National Science Foundation.
} 


\section{Introduction}

The use of electronic sensors with wireless communication capacity to monitor environmental phenomena is increasing $[1,2,3]$. In most of these systems there is rarely a need for device addressing beyond a small locality (e.g., temperature of a region or location of a tracked object). However, with the decreasing cost of wireless sensor network devices and the increasing number of sensing capabilities that a single device can exhibit, a deployed sensor network has resources that can fulfill many functions and overlaid missions. For example, a single mote can sense light, relative humidity, temperature, pressure and has a two-axis accelerometer [4]. Increasingly this sensing capability is moving to handle the larger complexity of audio and video data [5].

We envision these many-functioned capabilities of the sensor nodes not strictly as a baseline for building specific sensing applications but rather as serving multiple, overlaid missions involving possibly distinct tasks. One could imagine these missions as co-located or collaborative, potentially performing integration of data originating from different locations or functions of the sensor network. An example of this kind of integration might be the correlation of habitat weather data with the tracking of animals.

Characteristic of this setting is the sometimes independent, sometimes dependent collection, dissemination, and consumption of data originating from diverse sensor devices in the network. Consumers are expected to be applications, classes of users, and individual users each with different data requirements and communication needs. Under these circumstances, a deployed sensor network can be subject to a high inquiry arrival rate (due to being able to collect varied sets of data that are of interest to different user communities), in which each inquiry requests data from only a subset of the sensor nodes (reflecting different areas of interest from the users of the sensor network). In this scenario, flooding mechanisms such as Directed Diffusion [6] are inefficient because each inquiry can trigger a flooding to the whole network, even when only a subset of sensors are relevant to the inquiry.

To address this issue, we propose establishing a hierarchy of attributes which form the basis of the most common inquiries, and in which an upper level attribute contains the attributes of a lower level. For example, the attribute "building" contains the "floor" attribute, which in turn contains an attribute "room." By clustering sensors according to such an attribute hierarchy, we can forward inquiries to only the relevant parts of the sensor network and thus save energy and reduce network traffic. In this paper we show algorithms that can cluster the sensors according to a pre-defined attribute hierarchy, support 
insertion and deletion of hierarchy levels, and implement clusterhead fault tolerance and load balancing mechanisms by rotating the clusterhead functionality among cluster members. We also demonstrate analytically that inquiry dissemination over such virtual hierarchy has significant bandwidth gains over a flooding-based scheme under the scenario considered.

The remainder of the paper is organized as follows. In Sec. 2 we describe related work. Our attribute hierarchy representation and our clustering algorithms are presented in Sec. 3. In Sec. 4 we compare our hierarchical clustering scheme with a flooding scheme. We conclude in Sec. 5 .

\section{Related Work}

Node clustering techniques are significant for our approach and much work exists $[7,8,9$, 10, 11, 6, 12]. Lin and Gerla [7] select clusterheads based on node ID, while Ramanathan and Steenstrup [8] propose forming clusters based on link quality. Clustering is proposed in both cases to provide scalability and service guarantees. Admission control and bandwidth allocation are all performed within the cluster. Amis et al. [9] propose an election algorithm that chooses clusterheads in such a way that these form a dominating set. Moreover, nodes are guaranteed to be at most $d$ hops away from a clusterhead. McDonald and Znati [13] propose to form clusters in order to offer probabilistic bounds on path availability. The path availability model is built on top of a mobility model that is presented in the same paper. Banerjee and Khuller [10] proposed algorithms that form and maintain a hierarchical set of clusters under mobility. The clusters formed satisfy certain design objectives, such as nodes belonging to a constant number of clusters at one hierarchy level, low overlap between two clusters, etc. Ramachandran et al. [11] propose algorithms that form star shaped clusters at a pre-defined maximum size, with the Bluetooth [14] model in mind. Estrin et al. [6] proposed a clustering mechanism that can ensure bi-directional link connectivity for nodes in the network. Ghiasi et al. [12] propose an optimal $k$-clustering algorithm for sensor networks, in which $k$ clusterheads are selected and the clusters are balanced. It is shown that this problem is solved optimally using min-cost network flow.

The clustering algorithms above attempt formation of clusters that satisfy certain invariant properties (leaders have lowest ID), communication metrics (link quality, bi-connectivity) or topological properties (maximum cluster radius, balanced clusters, path availability, etc.). Our algorithms form clusters that reflect possible traffic patterns. By tying attributes that are relevant to inquiries posed to the sensor network to the overlaid cluster structure, we are 
establishing clusters that reflect application level communication needs rather than network level topological criteria.

Clustering algorithms that satisfy application level communication requirements have also been proposed $[15,16,17]$. Clusterheads in LEACH (Low Energy Adaptive Clustering Hierarchy [15]) and HEED (Hybrid Energy-Efficient Distributed Clustering [16]) are all elected through a randomized algorithm which guarantees that the role of a clusterhead is shared by all available nodes. HEED specifically uses residual energy in clusterhead election. Bandyopadhyay and Coyle [17] use stochastic geometry to derive an expression for the communication cost of cluster members to the clusterhead. From this expression the cluster radius and the probability of a node becoming a clusterhead is obtained. Our work differs from the above in that there is no support in their clustering algorithms (or architectures) to exploit biased communication patterns in sensor networks, which we believe will be evident if a large sensor network becomes a shared resource.

DataSpace [18] and SINA (Sensor Information Networking Architecture [19]) are additional relevant schemes for sensor networks. DataSpace [18] is a generalized geography-based (using GPS coordinates) routing architecture that can support querying and monitoring of objects in the DataSpace. It uses hierarchical data cubes (which represent 3D regions in space) and directory services in data cubes to achieve its goals. There is no reference to specific clustering mechanisms in this work. Clustering and attribute-based naming are both mentioned in SINA; however, the clustering algorithm is not tied to the attributes of sensors, and is proposed only to facilitate scalable operations. Our scheme is distinct in its ability to exploit traffic patterns via a hierarchy and the absence of reliance on GPS positioning coordinates.

Because our work supports delivering queries to relevant regions of the network, this can be seen as complementary to data-centric storage approaches, such as GHT (Geographic Hash Tables) [20], DIMENSIONS [21], DIFS (Distributed Index of Features in Sensor networks) [22], DIM (Distributed Index of Multi-dimensional data) [23] and Fractionally Cascaded Information (FCI) [24]. In GHT, DIM and DIFS the emphasis is in finding ways to store data in a predetermined way so that queries can efficiently find the necessary information. GHT and DIM use geographical hash functions that store data in pre-determined regions, while DIFS distributes data to multiple sensors according to the value of the data and the pre-defined spatial coverage each sensor has. This construction allows load balancing over index nodes and supports range queries as well. Our approach establishes hierarchies within the network and summaries of information so that queries may be routed to where the sensors containing 
the information are. Data is stored in the sensors detecting the event and not moved (nor replicated) in other sensors.

DIMENSIONS [21] advocates aggregating data hierarchically by clusterheads and using wavelet transforms to produce multi-resolution views of the network. Thus a query that did not require full resolution view of the data could be answered at a higher hierarchy level. Gao et al. [24] makes a similar argument and propose that sensors should only know a fraction of the information from distant parts of the network. They partition the network by using a quadtree structure, and sensors have different views of the network when considered at different levels in the quadtree. In this structured format, queries are bounded in complexity, as the authors show in their paper. In the two schemes above clusters form independently of the content of the sensors or the frequency and "shape" of queried regions. In contrast, our clusters are formed essentially based on the attributes queried and relevant regions.

Our work closely resembles Semantic Routing Trees (SRT) [25]. SRT proposes overlaying a tree on the sensor network, in which sensors track the value of a single attribute. Parent sensors know the value range of the attribute of all of its descendant sensors, and forward queries to a child only if it and its descendants can answer the query. A generalized approach to content based networking is CBCB (Combined Broadcast and Content-Based routing) [26]. $\mathrm{CBCB}$ assumes the existence of a broadcast layer that reaches all nodes in the network. In [26] nodes broadcast their predicates, i.e., a set of constraints over the attributes, along the broadcast tree. Matching data is attracted and forwarded to the nodes issuing the predicates. Nodes along the broadcast tree track the predicates issued and only forward relevant data that has been requested. Our work does not attempt to track query routing at every node in the network, instead, we form attribute equivalent clusters of sensors and use these clusters to route queries to relevant sensors. By changing how such clusters are formed, i.e., by adding or removing specific nodes in the attribute hierarchy, we can determine the granularity of control we desire in the query propagation and thus achieve higher gains by avoiding redundant traffic in the network.

\section{Attribute Based Hierarchical Clustering}

Because most sensors in a sensor network are intended to monitor phenomena and report results elsewhere, they can be collectively modeled as a large spatially distributed database [18, 27]. Examples of inquiries (information requests) that might be posed include: 
- How many nests in the northeast section of the forest currently have birds in them?

- What is the average temperature in the laboratories in the basement of building 10 ?

- Detect congestion in the intersection of Main and Broadway and control traffic lights to relieve the congestion.

- What is the frequency of vibration at 12:00?

If we relied on data flooding to disseminate the inquiries within the sensor network, all sensors would be affected whenever an inquiry requested a different type of data, which can be energy inefficient. To save energy, we propose clustering the sensors according to attributes that are meaningful to the inquiries and that can be exploited to reduce unnecessary traffic. One candidate that fulfills the requirements is to establish hierarchies of attributes that are location based, and in which upper level hierarchies contain lower level hierarchies. By location we mean attributes that are spatially related and by containment we imply that sensors that share a common lower level attribute automatically share all upper level hierarchy attributes.

We choose the location attribute as the clustering criterion for several reasons: (1) location attributes are general enough to be used in most environments ("geographical section" clusters for sensors covering a national park, "room" clusters inside a building, etc.); (2) hierarchies can also be easily built (room $\subset$ floor $\subset$ building, etc.); (3) the containment of lower level clusters by higher level ones allows us finer control over the selection of sensors that will receive an inquiry; and (4) it is easier to implement adaptive schemes which may go back and forth between pure flooding schemes, which is the same as having only one cluster containing all sensors, and hierarchically clustered schemes, depending on the dynamic cost effectiveness analysis. The process of designing and specifying the hierarchies is beyond the scope of this paper. We assume the existence of an attribute hierarchy, which we interchangeably call "containment hierarchy" $(\mathrm{CH})$.

In the presence of these hierarchical clusters, lower level clusterheads collect cluster member information into "catalogs" and send them to their upper level clusterheads. When inquiries arrive, they are processed and relayed by the top level clusterhead to the lower level clusterheads according to the catalog information possessed. Only when the inquiries arrive at the relevant clusters are they flooded to all the sensors in the clusters. Such location based containment hierarchies map themselves naturally to many scenarios (buildings, geographical areas) and can be represented as directed acyclic graphs (DAGs), as can be seen from the examples in Fig. 1. We call such DAGs Containment-DAGs or C-DAGs for short. 

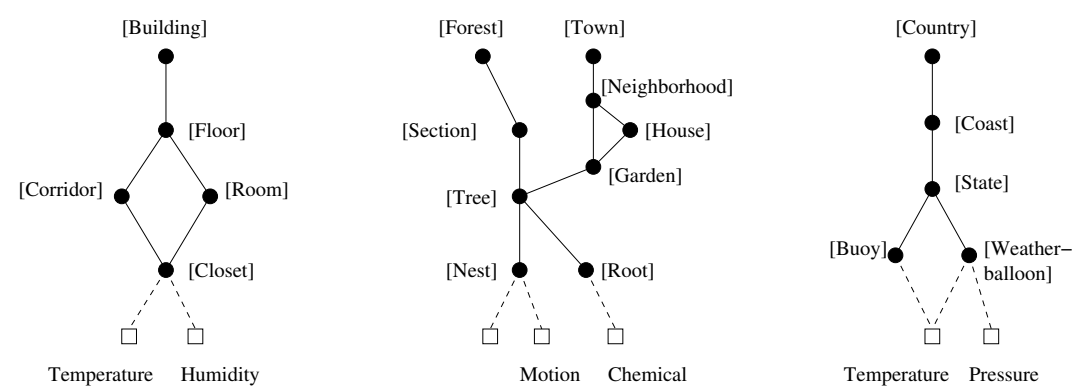

Figure 1: Examples of Attribute Containment Hierarchies

In Fig. 1 we show three examples of containment DAGs (C-DAGs). Nodes in black represent attributes that are relevant for users of the sensor network. White boxes represent types of data that can be collected by deployed sensors. Thus the leftmost C-DAG can be used to collect information regarding temperature and humidity conditions in a building, while the rightmost C-DAG can be used for temperature and pressure sensors monitoring weather conditions along the coast. In the center C-DAG of Fig. 1 we show an example of two attribute hierarchies (for "Forest" and for "Town") that have a common relevant attribute ("Tree"). In the figure, sensors with the same room number automatically share the same floor number. Sensors in the same garden have to be in the same neighborhood.

Our current work applies mainly to static or almost static sensor networks, as represented by habitat [2], traffic [3] or structural integrity monitoring applications [1], and by sensor network fields deployed for target classification and tracking [28]. It is possible to support non-location based clusters (e.g., sensors belonging to the same "family") by forming initially a location-based attribute hierarchy and establishing registration and update mechanisms to cope with physical distance and/or mobility. This is however reserved for future work. We present next our clustering algorithm.

\subsection{Attribute Based Clustering}

The algorithms we developed form same-attribute clusters with one clusterhead and rotate the clusterhead functionality among cluster members. Clusterheads will also gather information regarding their cluster members so as to be able to decide whether to flood or drop inquiries that reach them. Cluster sizes are constrained whenever possible, so as to avoid managing disproportionately large clusters. Devices with higher energy levels are selected in the clusterhead rotation process. Unicast routes are established among adjacent level clusterheads in the process to facilitate any future information exchange. In addition the algorithms detect and recover from clusterhead failures and support dynamic membership updates, effectively 
allowing dynamic $\mathrm{CH}$ level updates (i.e., the containment relationships may adapt to the types of inquiries). Specific parts of the algorithms are presented below.

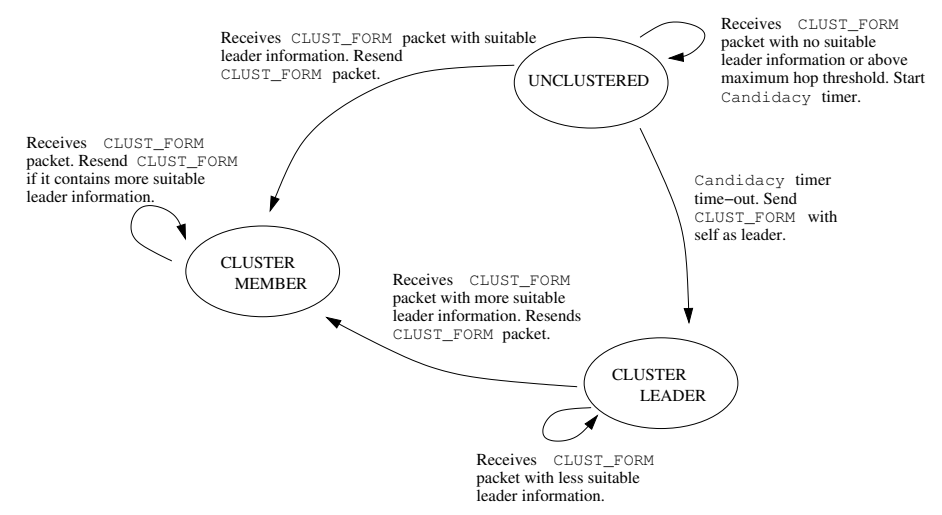

Figure 2: Finite State Machine for Cluster Formation

Cluster Formation We propose a modified leader algorithm [29] to form clusters (thus we will call clusterheads cluster leaders interchangeably) by attribute values and potentially limited by hop-count. Fig. 2 describes the finite state machine of our cluster formation algorithm $^{2}$. All cluster formation decisions are localized and all clusters across all hierarchy levels are formed in one network-wide flooding. This flooding is part of the maintenance cost which is independent of the inquiry arrival rate, and which is "amortized" as this clustered structure is re-used to deliver new incoming inquiries. Our cluster formation algorithm can be summarized as follows:

- One device (e.g., a base-station) starts the clustering process by broadcasting a cluster formation packet CLUST_FORM. Devices which hear this packet wait for a random amount of time (based on their energy levels [6]) and then announce their intention of becoming candidates through a broadcast (they attach their energy level information in this broadcast).

- The device with highest energy broadcasts its candidacy packet first. Leader candidates which hear such a broadcast cancel their timers and rebroadcast the higher energy leader's candidacy packet with hop count increased by one. Ties are broken by deterministic methods (i.e., lowest ID). The same packets received more than once are dropped.

- Devices which had selected leaders but which hear more suitable leader candidates switch leaders and rebroadcast the new leader's candidacy packet.

\footnotetext{
${ }^{2}$ For clarity, in the finite state machines of Fig. 2 - Fig. 5 steps to handle catalog collection and exchange are absent since these are not essential for understanding the clustering aspects of the algorithms.
} 
- When a device hears a cluster formation packet from a neighbor device which has a different attribute value in one of its $\mathrm{CH}$ levels (e.g., sensor 23 in room 445 hears from a sensor in room 442), it will try to become a leader candidate of the region with new attribute value (sensor 23 becomes leader candidate for room 445).

- Devices keep track of the hop count to the leader they are selecting and the neighbor devices through which they heard the packet. If it exceeds a pre-defined $\mathrm{CH}$ level threshold value, then the device will become a leader candidate and form a new cluster within the same attribute value region (thus room 445 may have more than one cluster). We call this hop-count based new cluster formation.

- Cluster leaders at the lowest CH level wait for a specified time interval before flooding (within the cluster) a request for cluster member information from its members. All cluster leaders wait a time out period (proportional to the cluster hop-threshold number, collect any member related information into a "catalog" and forward a summary of the information they collected to their higher level leader. This is so that top level leaders can make informed decisions on whether to forward or drop an arriving inquiry.

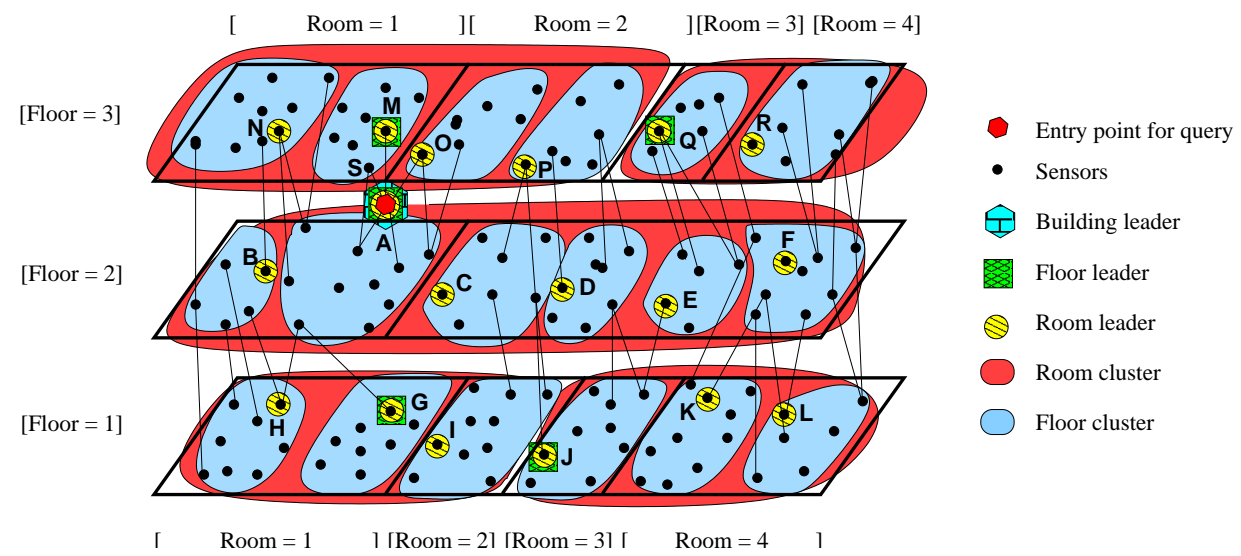

Figure 3: Attribute Containment-Based Clustering.

Note that clustering happens simultaneously across all $\mathrm{CH}$ levels. Thus our clustering scheme requires only one network-wide broadcast for the formation of the clusters at all $\mathrm{CH}$ levels. Although we apply node energy level as an attribute for leader selection, this is is not intrinsic to the algorithm and is not limiting. Any function of a sensor's attributes can be used for leadership candidacy. The hop-count based new cluster formation rule is overridden when there is attribute change in a lower level $\mathrm{CH}$ value. If there is no lower level, then new clusters may be formed as soon as the hop-count limit is reached. This is to avoid having different clusters in the same room answer to different floor leaders. 
We show an example of our clustering algorithm in Fig. 3. Cluster formation starts from node $A$, which elects itself as building, floor, and room leader. When it broadcasts the cluster formation packet, node $M$ accepts $A$ 's building leadership, but notices that the packet came from a different floor and room, and elects itself as leader of its floor and its room. Upon $M$ 's broadcast, node $O$ accepts $M$ 's floor leadership, but keeps its own room leadership candidacy and eventually becomes room leader. Node $S$ accepts leadership from $A$ and $M$, canceling any candidacy timers it may have. As cluster formation packet propagates, new room clusters are formed if the rooms are large (e.g., rooms 1 and 2 on floor 3) but since different floor clusters cannot be formed in the same room, there is only one floor cluster on floor 2. On floor 1 , nodes $G$ and $H$ both broadcast their floor candidacy close to one another, but $G$ is the "most suitable" leader because we used the lowest id function as a tie breaker. Node $H$ remained room leader because of the hop distance between itself and $G$. The building cluster encompassing all sensors has not been shown for sake of clarity.

Cluster Leader Rotation Leader rotation avoids single devices from being completely energy-depleted due to their burden in the clusterhead role. The rotation period is adjusted according to the frequency of inquiries arriving at the cluster and to the leader's level in the hierarchy level (higher level leaders rotate less). The steps in our algorithm are:

1. After a time-out interval, the sensor with the highest energy left in the cluster floods the cluster announcing its leadership candidacy, establishing a routing tree rooted at itself;

2. If multiple candidacies are heard, the "most suitable" (determined through a localized decision) is selected;

3. The old leader, upon time-out, unicasts its catalog information to the newly elected leader via the routing tree, and the new leader sends a catalog information update to its higher level leader. This update establishes the unicast route from the new leader to the higher level leader.

Fig. 4(a) shows the finite state machine of the rotation algorithm with the characteristics listed above.

Cluster Recovery Algorithms Cluster leaders send periodic LEADER_ALIVE messages to its $k$-hop neighbors ( $k$ being a tunable parameter of the algorithm). These neighbors also keep a copy of whatever information the cluster leader is maintaining. The neighbor which detects cluster leader failure floods the cluster identifying itself as "interim leader" 

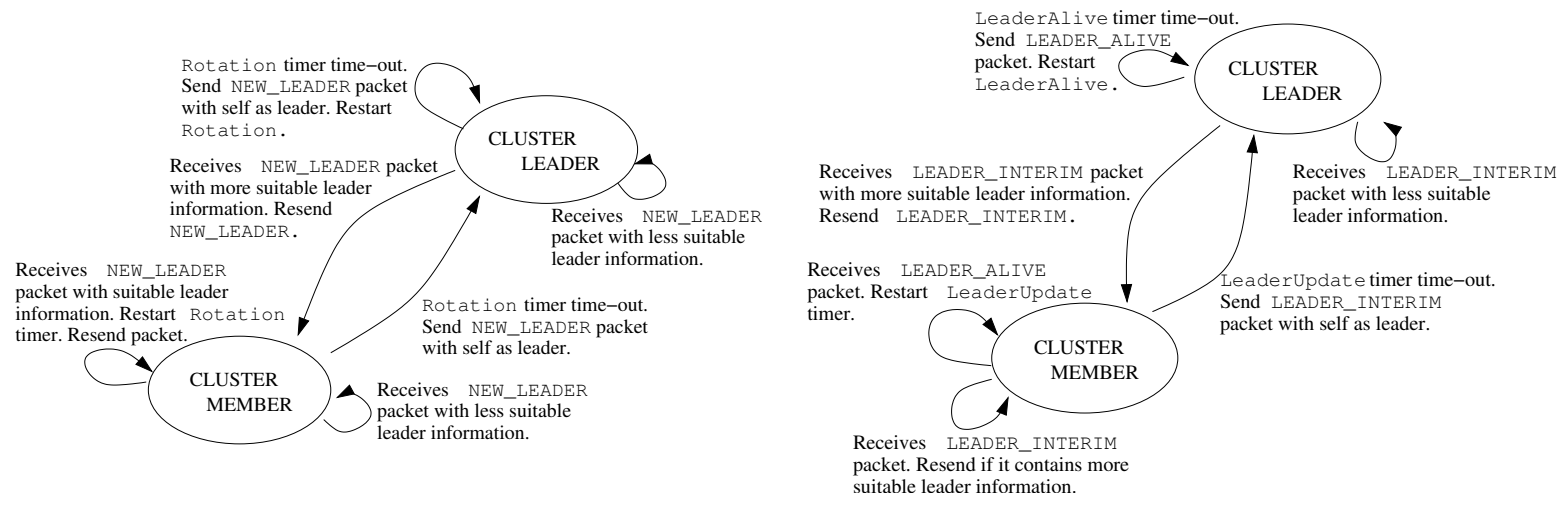

Figure 4: Finite State Machine for (a) Leader Rotation and (b) Leader LEADER_ALIVE Packet Exchange with $k$-hop Neighbors

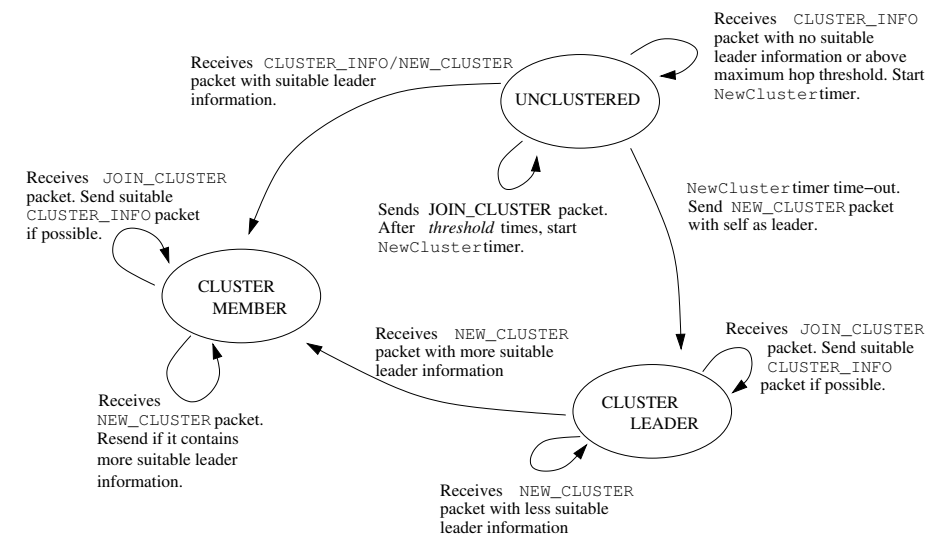

Figure 5: Finite State Machine for joining existing clusters

(see Fig. 4(b)) and a rotation mechanism follows. Cluster member failures do not trigger any recovery mechanisms, for we assume the sensor network to be dense enough, in which individual sensor failures do not impair cluster related functions and properties.

If the network is not large or not dense enough, then peer monitoring among sameattribute leaders may be necessary to recover from partitions in the attribute value region. For example, consider the case in which a sensor in room 445 fails and breaks one cluster into two partitions, but both are reachable through sensors along the corridor. In these instances, the partition without a leader will detect soon that no leader rotation packets have traversed it. After a fixed time-out value plus a random interval of time one of the sensors in the partition will broadcast an attribute-limited cluster formation packet and a leader candidacy packet, attempting to form a new cluster. After the new leader is established it will collect catalog information from its members and contact its immediately higher level leader. 
Cluster Join and CH Update Algorithms Newly deployed sensors will attempt to join the "best" neighboring clusters that have the same attribute values. They do so by broadcasting a request for membership packet. If no answer is received for $n$ such broadcasts (with exponentially increasing intervals) then the sensor remains isolated and will cluster only when a cluster formation packet arrives. Thus all initial sensors are isolated until "triggered" by an external signal from their base-station.

However, if there are clustered sensors nearby, they will answer the membership request by sending their $\mathrm{CH}$ instance information, as well as all of their $\mathrm{CH}$ cluster information. The new sensor may join the "best" cluster (at each $\mathrm{CH}$ level), if attributes match, or may attempt to form a new cluster. Fig. 5 shows the finite state machine for cluster join and update algorithms.

This mechanism effectively supports dynamic $\mathrm{CH}$ updates. If there is an addition of a $\mathrm{CH}$ level, then sensors receiving the $\mathrm{CH}$ update are effectively "new without-leader" (in that level) sensors which are in an already deployed network. They will request membership but will receive cluster information without any matching $\mathrm{CH}$ level instance, at which point they will group themselves together and elect new leaders. These new leaders will contact (potentially through flooding the higher level cluster) their higher level leaders and lower level leaders (if they exist) and re-establish the unicast communication architecture among adjacent level clusterheads.

To complete our discussion of dynamic $\mathrm{CH}$ updates, note that the removal of a level does not affect any member, since sensors kept all information for all $\mathrm{CH}$ levels. They simply erase the information regarding that level. Leaders of the level below the removed one send catalog update information to leaders two levels up in the old $\mathrm{CH}$ (such paths were formed when the higher level leaders were elected). In the next section we present a communication cost analysis of our hierarchical clustering mechanism and of flooding based schemes.

\section{Cost Analysis of Containment Hierarchy and Flooding Techniques}

In this section we present an analysis to establish the effectiveness of creating and maintaining CHs over the lifetime of a sensor network as compared to a flooding-based scheme. We focus on the communication cost for the dissemination of inquiries since power consumption in a sensor node is dominated by radio communication [25]. 
Preliminary Considerations The interaction of a community of users with a deployed sensor network can be represented as inquiries that arrive to the sensor network with a certain rate $\lambda$. Each arriving inquiry affects a portion $Q$ of the sensors in the network according to a probability distribution function $P_{Q}$. The set of all possible portions $Q$ is denoted $\mathbf{S}$. We make the following simplifications before proceeding to some theoretical analysis:

1. We assume that the cost of "tagging" the sensors so that they become aware of their attributes is the same for both schemes;

2. The arrival rate $\lambda$ represents the rate of arrival of requests for data of a type not queried previously and/or from sensors of a different attribute, i.e., requests that trigger a flooding in the flooding-based schemes. As stated previously, our scenario is consisted of a network of multi-modal sensors. This network is a shared resource, and its users are members from diverse research communities. The arrival $\lambda$ models the multiple inquiries that are initiated by this aggregate pool of users.

3. We assume that answers to inquiries traverse through paths formed during inquiry propagation, and such paths form an inverted tree structure. The exact number of transmissions needed to send the collected data back is dependent on the tree structure of each scheme (CHs and flooding), and is left for future research. However, since the underlying mechanism is the same (tree structures), we believe that the order of magnitude of the number the transmissions in both cases is similar.

4. The cost we compute is that of the number of transmissions required to deliver the inquiry. Although the cost of listening cannot be neglected, the analysis we perform here is between our scheme and flooding schemes. In the absence of different scheduling mechanisms, counting the number of transmissions yields the same estimate of power consumption in both schemes (i.e., in both schemes the same number of sensors will be listening at each transmission).

We will next derive some quantitative cost comparison results between $\mathrm{CH}$ and flooding based schemes.

\subsection{Analytical Results}

Flooding Costs In a flooding based scheme, when a new inquiry arrives, it is flooded to the whole network. In such schemes, a wireless network composed of $N$ sensors, deployed 
over total time epoch $T$, incurs the following expected cost Cost $_{\text {Flood }}$ for inquiry delivery:

$$
\text { Cost }_{\text {Flood }}=\lambda T N
$$

A scheme that actively maintains a $\mathrm{CH}$ structure $L$ on top of the sensor network will have two cost components: a maintenance cost $\operatorname{Cost}_{C H}^{(m n t)}$ and an inquiry dependent cost $\operatorname{Cost}_{C H}^{(i n q)}$. The maintenance cost involves communication costs needed to establish hierarchies, clusters, message exchanges between clusterheads for coordination and catalog information dissemination for inquiry forwarding. Note, however, that such maintenance cost is inquiry independent, i.e., it does not increase with the frequency of new inquiries. The inquiry dependent cost $\operatorname{Cost}_{C H}^{(i n q)}$ is the cost incurred in forwarding the inquiry to only the relevant parts of the network, based on the hierarchical structure $L$. In order to compare Cost $_{F l o o d}$ and Cost $_{C H}$, we will study an example scenario and derive analytical expressions for Cost $_{C H}$ and compare it with Eq. 1.

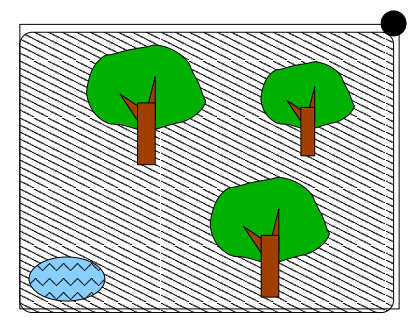

(a) Flat Structure (1 Level)

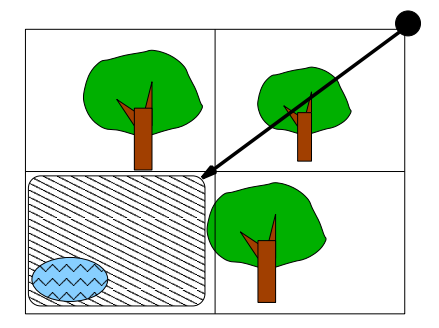

(b) 2 Level Structure

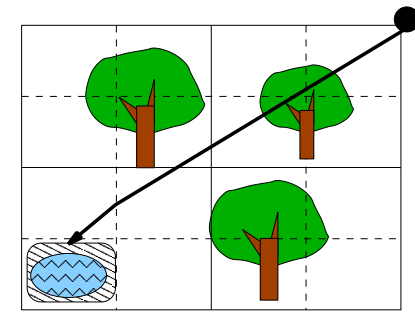

(c) 3 Level Structure

Lake (Region of Interest)

Sensors Affected

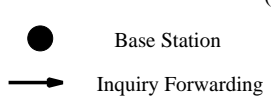

Figure 6: Inquiries are propagated differently in a sensor network depending on the existence of hierarchy levels: (a) contains one hierarchy level, (b) two hierarchy levels, and (c) three hierarchy levels.

Consider now Fig. 6. In the left-most part (Fig. 6(a)) there is only a single-level flat network. The communication costs associated with inquiry delivery in this flat network and flooding based schemes is equivalent. In this case there is no hierarchy maintenance costs. However, when an inquiry for the lake arrives, even though only sensors in the lake need respond, still the inquiry reaches all sensors in the whole square area, since there is no mechanism to distinguish one sensor from another. In Fig. 6(b) we establish a hierarchy with one extra upper level (two levels total) and divide the area into four quadrants. In this case the same inquiry will affect only $1 / 4$ of the sensors of Fig. 6(a) plus sensors involved in forwarding the inquiry from the base station to the lower-left quadrant. In this case a maintenance cost exists to establish and preserve the structure of quadrants (i.e., establishing 
the clusters that map to the four quadrants, choosing clusterheads and maintaining load balancing schemes), as well as inquiry forwarding costs. In Fig. 6(c) we add another level. With this we reduce the number of sensors affected by the inquiry to only $1 / 16$ of those in Fig. 6(a) and to $1 / 4$ of those in Fig. 6(b). The trade-off in Fig. 6(c) is a higher maintenance cost for the two extra levels and a higher inquiry forwarding cost, if the region relevant to the inquiry is far from the base-station.

In our example, first the inquiry is forwarded from the point of entry (e.g., a base station) to the top level (level $=1$ ) leader. If the inquiry is for the whole network, the latter floods it, otherwise it forwards the inquiry to appropriate leaders at level $=2$ (with appropriate region attribute). These will likewise determine whether the inquiry is for their whole region, in which case they flood the region, or forward the inquiry to appropriate sub-region leaders (these will then flood their sub-region, and so on). The cost of flooding the network is $N$, while that of a region with level $=2$ is $N / 4$ and a sub-region with level $=3, N / 16$ etc. Unicasts from the base station to the top level leader have estimated cost of the order of $\sqrt{2 N}$ since there are as many hops in the longest path in the square area. Likewise, the cost estimate for forwarding the inquiry from a level 2 leader to a level 3 leader is of the order of $\frac{\sqrt{2 N}}{2}$.

Cost of Containment Hierarchy Maintenance CH scheme has an associated "maintenance cost" for the entire epoch due to the periodic rotation of clusterheads. Suppose the clusterhead rotation period at level $=i$ is $T_{i}$ for $i=1$ to $\ell_{\max }$. The total maintenance cost is then given by:

$$
\operatorname{Cost}_{C H}^{(m n t)}=N+2 N \sum_{i=1}^{\ell_{\max }} \frac{T}{T_{i}}+\sqrt{2 N} \sum_{i=2}^{\ell_{\max }} 2^{i} \frac{T}{T_{i}}
$$

Initial clustering involves one network-wide broadcast that contributes $N$ (first term in Eq. 2) to the cost since each node transmits a broadcast packet only once. The rest of the terms correspond to cluster maintenance costs. There are $\frac{T}{T_{i}}$ clusterhead rotations at level $=i$. Each rotation at level $=i$ requires one broadcast at that level followed by all sensors in the cluster responding to update the catalog information. The broadcast contributes $N$ to the cost at each level and so does the catalog update step. This accounts for the second term in Eq. 2. The third term corresponds to the unicast cost of communication of catalogs between cluster leaders, and is a simplification of $4 \sqrt{2 N} \frac{T}{T_{1}}+16 \frac{\sqrt{2 N}}{2} \frac{T}{T_{2}}+\cdots$.

Cost of Inquiry Dissemination Now, consider a model where one particular region at level $=\ell_{\max }$ receives an inquiry with probability $p$. For example, the region getting inquiry in Fig. 6(b) (we will henceforth refer to this region as R). For simplicity, we assume that 
inquiries involving the rest of the possible combinations are equiprobable with probability $q$, e.g., $q=\frac{1-p}{14}$ for $\ell_{\max }=2$. In this model, the average cost incurred for dissemination of inquiries over time $T$ is given by:

$$
\operatorname{Cost}_{C H}^{(i n q)}=\lambda T\left\{\sqrt{2 N}+\sum_{Q \in S} P_{Q} C_{Q}\right\}
$$

In Eq. 3 the estimated cost of forwarding an inquiry from the base station to the top level leader is of the order of $\sqrt{2 N}$. This analysis assumes the presence of one leader per attribute-value region. The second term expresses the cost of disseminating the inquiry to its intended destinations while using the constructed hierarchy. The summation occurs over all elements $Q$ in the set $S$ of all possible combinations of sub-regions in the sensor network. In general there are $s=4^{\ell_{\max }-1}$ sub-regions and hence $|S|=2^{s}-1$. $P_{Q}$ is the probability of an inquiry involving the particular combination of sub-regions $Q$ from the set $S$ and $C_{Q}$ is the cost of disseminating that particular style of inquiry. If $Q$ spans all sub-regions in the network (level $=1)$, then $C_{Q}=N$; if it only spans $m<4$ subregions at level $=2$, then $C_{Q}=m\left(\sqrt{2 N}+\frac{N}{4}\right)$. If $Q$ involves $m$ sub-regions $r_{1}, r_{2}, \ldots, r_{m}$ at level $=2$ and also involves specific subregions inside each of these $r_{k}$ 's at level $=3$ (say, $\left\{r_{11}, \ldots, r_{1 n_{1}} ; r_{21}, \ldots, r_{2 n_{2}} ; \cdots ; r_{m 1}, \ldots, r_{m n_{m}}\right\}$, then the cost is given by:

$$
C_{Q}=\sum_{k=1}^{m}\left\{\sqrt{2 N}+n_{k}\left(\frac{\sqrt{2 N}}{2}+\frac{N}{16}\right)\right\}
$$

The $C_{Q}$ term for a general level $i \leq \ell_{\max }$ can be expressed similarly as a sum of costs due to unicast and scoped broadcast within attribute sub-regions as have been illustrated above (not presented here). For $\ell_{\max }=2$ the total average cost incurred for dissemination of inquiries for the epoch $T$ is given by:

$$
\begin{aligned}
\operatorname{Cost}_{C H}^{(i n q)=} & \lambda T\left\{p\left(\sqrt{2 N}+\frac{N}{4}\right)+\right. \\
& \frac{3}{14}(1-p)\left(\sqrt{2 N}+\frac{N}{4}\right)+ \\
& \frac{6}{14}(1-p)\left(2 \sqrt{2 N}+\frac{N}{2}\right)+ \\
& \frac{4}{14}(1-p)\left(3 \sqrt{2 N}+\frac{3 N}{4}\right)+ \\
& (1-p) N+\sqrt{2 N}\}
\end{aligned}
$$

The total communication cost corresponding to our CH-based scheme is given by:

$$
\operatorname{Cost}_{C H}=\operatorname{Cost}_{C H}^{(m n t)}+\operatorname{Cost}_{C H}^{(i n q)}
$$



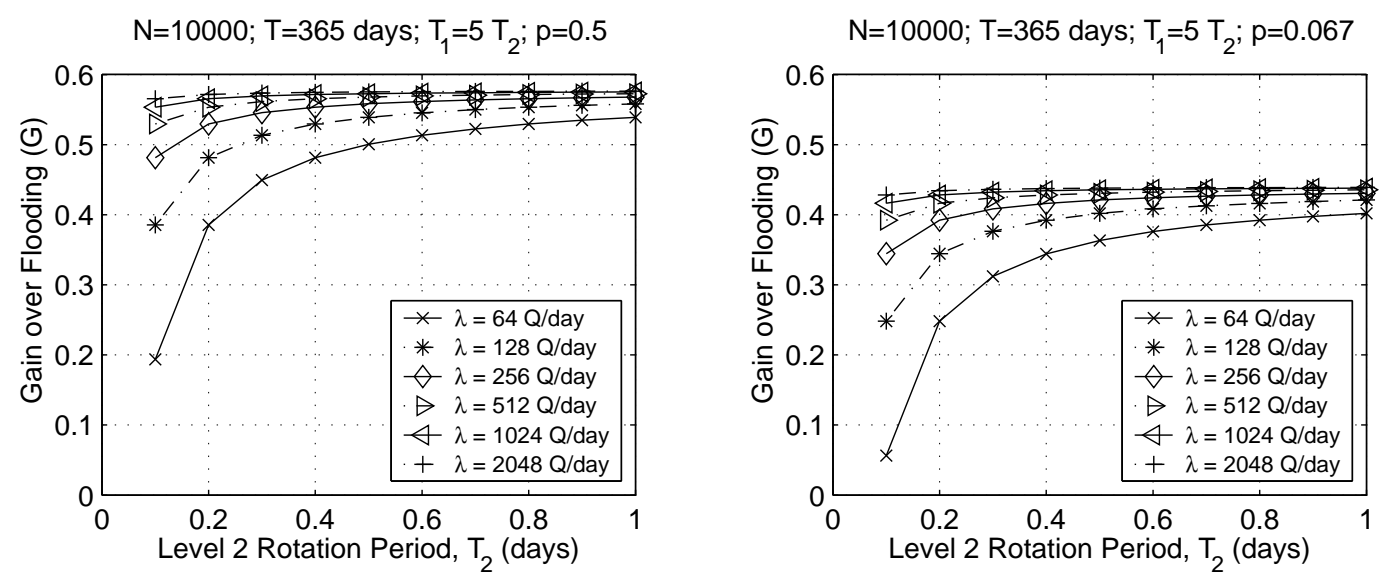

Figure 7: Effect of Rate of Inquiry and Clusterhead Rotation Period on Gains: Two levels in the Containment Hierarchy
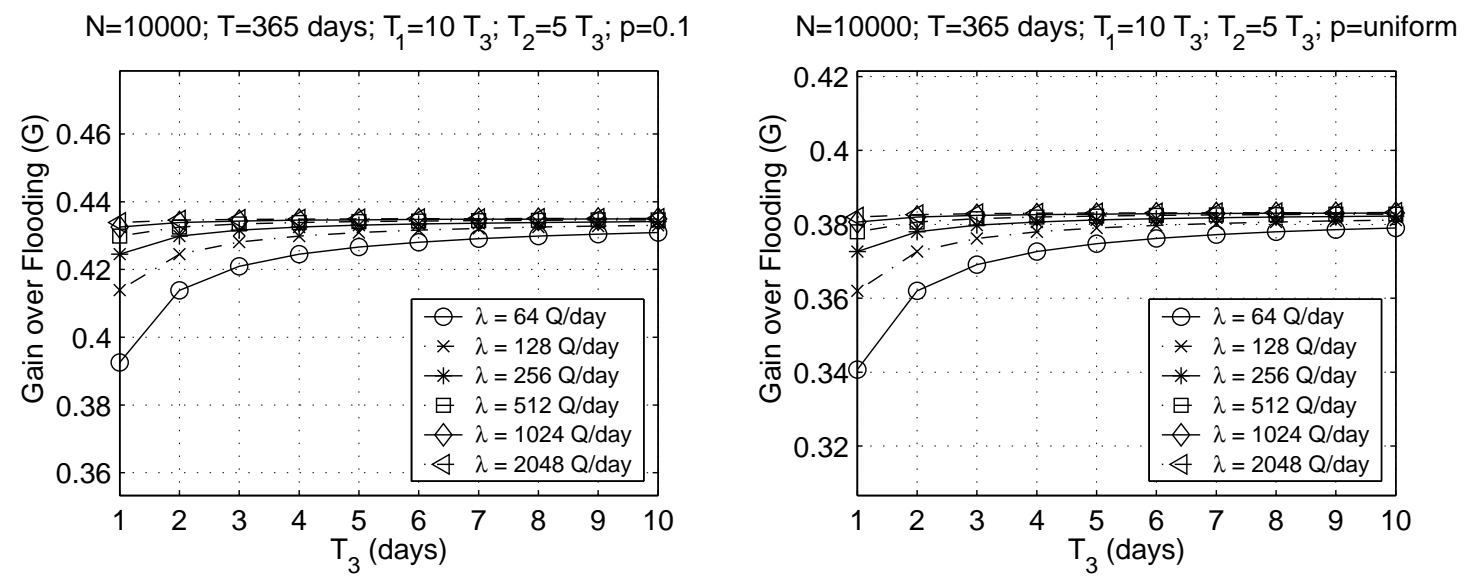

Figure 8: Effect of Rate of Inquiry and Clusterhead Rotation Period on Gains: Three Levels in the Containment Hierarchy

The first term of Eq. 5 corresponds to the case where region $\mathrm{R}$ gets a unicast inquiry ( $R$ 's cluster leader receives it from the level-1 leader; this incurs a worst case communication cost of $\sqrt{2 N}$ ) and then that is then disseminated by a broadcast within the $\frac{N}{4}$ sensors in R. Other terms in Eq. 5 correspond to various possible combinations of regions featured in the inquiries can be calculated using similar analysis. We do not explain each case in detail due to paucity of space.

We define our performance index, $G$, by:

$$
\begin{aligned}
G & =\frac{\text { Cost }_{\text {Flood }}-\text { Cost }_{C H}}{\text { Cost }_{\text {Flood }}} \\
& =\frac{\text { Cost }_{\text {Flood }}-\text { Cost }_{\text {CH }}^{(\text {mnt })}-\text { Cost }_{C H}^{(i n q)}}{\text { Cost }_{\text {Flood }}}
\end{aligned}
$$


Current sensor technology such as Mica motes have a lifetime in the range of approximately 6 months using AA batteries and a duty cycle of $2 \%$ (between active and sleep modes) [25]. The lifetime and energy efficiency of such sensors are likely to increase in the near future. In this analysis we assume an operating life of one year. In general, $\mathrm{CH}$ tend to outperform flooding-based schemes for larger time epochs due to amortization of the clustering cost.

First we study the case in which inquiries for one sub-region are extremely popular $(p=0.5)$. Results for this case are shown in Fig. 7(a). We see that as $\lambda$ increases, the dependence of $G$ over the the rotation periods $T_{1}, T_{2}$ diminishes. This is expected as $T_{1}, T_{2}$ influence the fixed maintenance cost due to attribute based clustering - as more inquiries arrive into the sensor network, the fixed cost penalty almost vanishes. In Fig. 7(b) we study the case in which all 15 combinations of regions are equiprobable $\left(p=\frac{1}{15}\right)$. We see similar behavior except that the gains are slightly lower in this situation. This is also expected because more possible destinations for the inquiries correspond to greater unicast costs in its dissemination. Similar results have been shown for the case of three C-DAG levels (corresponding to the scenario shown in Fig. 6(c)) in Fig. 8.

One interesting phenomena that can be observed from these curves is that the gains stabilize after $\lambda$ is increased past a certain value for every value of $p$. This is because for high $\lambda$ the contribution of $\operatorname{Cost}_{C H}^{(m n t)}$ towards $G$ is minimal after a certain threshold even for frequent rotation periods. The dominant contributor to the cost is thus $\frac{\operatorname{Cost}_{C H}^{(i n q)}}{\lambda T N}$ which is primarily linear in $p$ for large $N$. For this reason we observe different asymptotic values of $G$ as $p$ is varied.

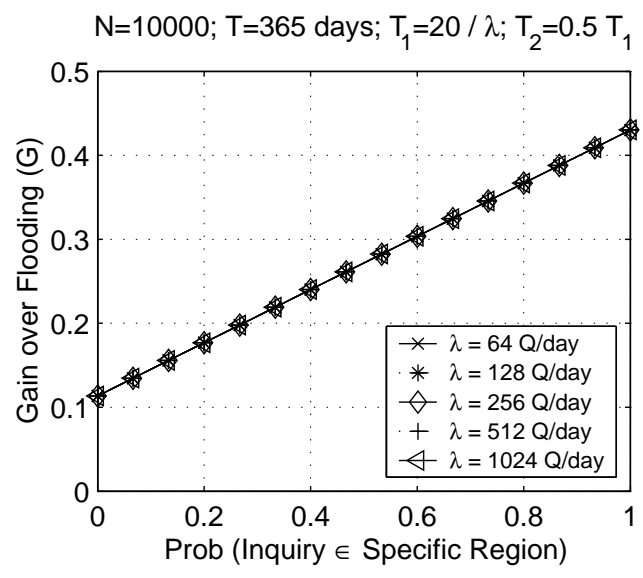

Figure 9: Gain vs. Probability for Proportional Rotation Periods: Two levels in C-DAG

If rotation periods $T_{i}$ 's are made inversely proportional to the mean arrival rates, e.g., 
$T_{i}=\frac{a_{i}}{\lambda}$, then Eq. 7 becomes:

$$
\begin{aligned}
G= & 1-\left(\frac{1}{\lambda T}+2 \sum_{i=1}^{\ell_{\max }} \frac{1}{a_{i}}+\sqrt{\frac{2}{N}} \sum_{i=2}^{\ell_{\max }} \frac{2^{i}}{a_{i}}\right)- \\
& \left(\sqrt{\frac{2}{N}}+\frac{1}{N} \sum_{Q \in S} P_{Q} C_{Q}\right)
\end{aligned}
$$

In this case gain $\mathrm{G}$ essentially becomes independent of $\lambda$ and linearly increases with probability p. This can be seen in Fig. 9.

In this section, we demonstrated that $\mathrm{CH}$ schemes yield gains over flooding-based schemes when there are sub-regions in the sensor network that are more targeted than others, i.e., when the distribution of inquiries is not uniformly distributed over time and space. We also showed that with increase in inquiry rate $\lambda$, $\mathrm{CH}$ schemes perform better since their structures can be re-used and are more directed towards specific target regions, whereas in a flooding-based scheme, a network-wide broadcast is necessary for each inquiry.

\section{Conclusion}

In this paper we showed a cost analysis of flooding based mechanisms for triggering data collection versus mechanisms which actively maintain a hierarchical structure to deliver inquiries. We show that under heavy utilization and high degree of sharing among a large community, sensor networks employing pure flooding systems are expected to be less efficient when compared to an attribute-based hierarchical system.

We propose an algorithm which is capable of supporting such hierarchies. This algorithm is our main contribution. It enables hierarchical clustering of sensors based on common attributes, and supports dynamic addition and removal of levels in the hierarchy. In addition, our algorithm is fast, requiring only one network wide flooding to establish all clusters across all hierarchy levels. It is also robust with respect to clusterhead failure, and implements load balancing by rotating the clusterhead functionality among cluster members.

\section{References}

[1] J. Paek, N. Kothari, K. Chintalapudi, S. Rangwala, N. Xu, J. Caffrey, R. Govindan, S. Masri, J. Wallace, and D. Whang, "The Performance of a Wireless Sensor Network 
for Structural Health Monitoring," in 2nd European Workshop on Wireless Sensor Networks, Istanbul, Turkey, Jan 31 - Feb 22005.

[2] A. Mainwaring, D. Culler, J. Polastre, R. Szewczyk, and J. Anderson, "Wireless Sensor Networks for Habitat Monitoring," in Proc. 1st ACM Intl. Workshop on Wireless Sensor Networks and Applications (WSNA'02), 2002, pp. 88-97.

[3] S. Coleri, S. Y. Cheung, and P. Varaiya, "Sensor Networks for Monitoring Traffic," in 42nd Annual Allerton Conference on Communication, Control, and Computing, U. of Illinois, September 2004.

[4] J. Hill and D. Culler, "MICA: A Wireless Platform For Deeply Embedded Networks," IEEE Micro, vol. 22, no. 6, pp. 12-24, Nov/Dec 2002.

[5] "Stargate Datasheet," URL, http://xbow.com/Products/Product_pdf_files/Wireless_ pdf/Stargate_Datash\%eet.pdf.

[6] D. Estrin, R. Govindan, J. Heidemann, and S. Kumar, "Next Century Challenges: Scalable Coordination in Sensor Networks," in Proc. 5th ACM MobiCom Conference, Seattle, WA, August 1999.

[7] C. R. Lin and M. Gerla, "Adaptive Clustering for Mobile Wireless Networks," IEEE J. Sel. Areas in Communication (JSAC), vol. 15, no. 7, September 1997.

[8] R. Ramanathan and M. Steenstrup, "Hierarchically-Organized Multihop Mobile Networks for Quality-of-service Support," ACM/Baltzer J. Mobile Networks and Applications, vol. 3, no. 2, August 1998.

[9] A. D. Amis, R. Prakash, T. Vuong, and D. Huynh, "Max-Min D-Cluster Formation in Wireless Ad Hoc Networks," in Proc. IEEE INFOCOM'00, Tel Aviv, March 2000.

[10] S. Banerjee and S. Khuller, "A Clustering Scheme for Hierarchical Control in Multihop Wireless Networks," in Proc. IEEE INFOCOM'01, Anchorage, Alaska, USA, April 2001.

[11] L. Ramachandran, M. Kapoor, A. Sarkar, and A. Aggarwal, "Clustering Algorithms for Wireless Ad Hoc Networks," in Proc. 4th Intl. workshop on Discrete Algorithms and Methods for Mobile Computing and Communications (DIALM'00), 2000.

[12] S. Ghiasi, A. Srivastava, X. Yang, and M. Sarrafzadeh, "Optimal Energy Aware Clustering in Sensor Networks," SENSORS Journal, vol. 2, no. 7, pp. 258-269, July 2002. 
[13] B. McDonald and T. F. Znati, "A Mobility-Based Framework for Adaptive Clustering in Wireless Ad Hoc Networks," IEEE J. Sel. Areas in Communication (JSAC), vol. 17, no. 8, August 1999.

[14] "Bluetooth Consortium," URL, http://www.bluetooth.com.

[15] W. Heinzelman, A. Chandrakasan, and H. Balakrishnan, "Energy-Efficient Communication Protocols for Wireless Microsensor Networks," in Hawaiian Intl. Conference on Systems Science (HICSS), Hawaii, January 2000.

[16] O. Younis and S. Fahmy, "HEED: A Hybrid, Energy-Efficient, Distributed Clustering Approach for Ad-hoc Sensor Networks," IEEE Transactions on Mobile Computing, vol. 3, no. 4, pp. 366-379, Oct-Dec 2004.

[17] S. Bandyopadhyay and E. Coyle, "An Energy Efficient Hierarchical Clustering Algorithm for Wireless Sensor Networks," in Proc. IEEE INFOCOM'03, 2003.

[18] T. Imielinski and S. Goel, "DataSpace - Querying and Monitoring Deeply Networked Collections in Physical Space," IEEE Personal Communications, October 2000, special Issue on Networking the Physical World.

[19] C. C. Shen, C. Srisathapornphat, and C. Jaikaeo, "Sensor Information Networking Architecture and Applications," IEEE Personal Communications, pp. 52-59, August 2001.

[20] S. Ratnasamy, B. Karp, L. Yin, F. Yu, D. Estrin, R. Govindan, and S. Shenker, "GHT: A Geographic Hash Table for Data-Centric Storage," in Proc. 1st ACM Intl. Workshop on Wireless Sensor Networks and Applications (WSNA'02), Atlanta, GA, September 2002.

[21] D. Ganesan, D. Estrin, and J. Heidemann, "DIMENSIONS: Why do we need a new Data Handling architecture for Sensor Networks?" in Proc. 1st Workshop on Hot Topics In Networks (HotNets-I), Princeton, NJ, October 2002.

[22] B. Greenstein, D. Estrin, R. Govindan, S. Ratnasamy, and S. Shenker, "DIFS: A Distributed Index for Features in Sensor Networks," in Proc. 1st IEEE Intl. Workshop on Sensor Network Protocols and Applications (SNPA'03), 2003.

[23] X. Li, Y. J. Kim, R. Govindan, and W. Hong, "Multi-dimensional Range Queries in Sensor Networks," in Proc. 1st ACM Conference on Embedded Networked Sensor Systems (Sensys'03), Los Angeles, CA, USA, November 2003. 
[24] J. Gao, L. J. Guibas, J. Hershberger, and L. Zhang, "Fractionally Cascaded Information in a Sensor Network," in Proc. 3rd Intl. Symposium on Information Processing in Sensor Networks (IPSN'04), 2004, pp. 311-319.

[25] S. Madden, M. Franklin, J. Hellerstein, and W. Hong, "The Design of an Acquisitional Query Processor for Sensor Networks," in Proc. ACM SIGMOD, San Diego, CA, June. 2003.

[26] A. Carzaniga, M. J. Rutherford, and A. L. Wolf, "A Routing Scheme for Content-Based Networking," in Proc. IEEE INFOCOM'04, Hong Kong, China, 2004.

[27] P. Bonnet, J. E. Gehrke, and P. Seshadri, "Querying the Physical World," IEEE Personal Communications, vol. 7, no. 5, pp. 10-15, October 2000, special Issue on Smart Spaces and Environments.

[28] R. R. Brooks, P. Ramanathan, and A. M. Sayeed, "Distributed Target Classification and Tracking in Sensor Networks," Proc. of the IEEE, vol. 91, no. 8, August 2003.

[29] J. A. Hartigan, Clustering Algorithms. New York, NY: Wiley, 1975. 